\title{
A NeW STUdY TO FIND OUT THE BEST COMPUTATIONAL METHOD FOR SOLVING THE NONLINEAR EQUATION
}

\author{
Mir Md. Moheuddin ${ }^{1}$, Md. Jashim Uddin² and Md. Kowsher ${ }^{3 *}$ \\ ${ }^{1}$ Dept. of CSE, Atish Dipankar University of Science and Technology, Dhaka-1230, \\ Bangladesh. \\ ${ }^{2,3}$ Dept. of Applied Mathematics, Noakhali Science and Technology University, \\ Noakhali-3814, Bangladesh.
}

\begin{abstract}
The main purpose of this research is to find out the best method through iterative methods for solving the nonlinear equation. In this study, the four iterative methods are examined and emphasized to solve the nonlinear equations. From this method explained, the rate of convergence is demonstrated among the 1st degree based iterative methods. After that, the graphical development is established here with the help of the four iterative methods and these results are tested with various functions. An example of the algebraic equation is taken to exhibit the comparison of the approximate error among the methods. Moreover, two examples of the algebraic and transcendental equation are applied to verify the best method, as well as the level of errors, are shown graphically.
\end{abstract}

\section{KEYWORDS}

Newton-Raphson method, False Position method, Secant method, Bisection method, Nonlinear equations and Rate of convergence.

\section{INTRODUCTION}

In numerical analysis, the most appearing problem is to determine the equation's root in the form of $f(x)=0$ rapidly where the equations of the types of $f(x)=0$ is known as Algebraic or Transcendental according to as

$$
f(x)=a_{0} x^{n}+a_{1} X^{n-1}+\ldots .+a_{n-1} x+a_{n},(a \neq 0),
$$

where the expression of $f(x)$ being an algebraic or transcendental or a combination of both.If $f(x)$ $=0$

at the point $x=\alpha$, then $\alpha$ is acquainted as the root of the given equation if and only if $f(\alpha)=0$. The equations of $f(x)=0$ is an algebraic equation of degree $k$ such as $x^{3}-3 x^{2}+12=0,2 x^{3}-3 x-6=0$ are some kinds of algebraic equations of two and three degree whereas if $f(x)$ takes on small number of function including trigonometric, logarithmic and exponential etc then $f(x)=0$ is a Transcendental equations, for instance, $x \sin x+\cos x=0, \log x-x+3=0$ etc are the transcendental equations. In both cases, the coefficients are known as numerical equations if it is pure numbers. Besides, several degrees of algebraic functions are solved by well-known methods that are common to solve it. but problems are when we are trying to solve the higher degree or transcendental functions for which there are no direct methods of how to get the best solution easily. Using approximate methods are a facile way for these sorts of equations. Numerical 
methods are applied to impart formative solutions of the problems including nonlinear equations. Various methods for discovering the nonlinear equation's root exists. For example, the Graphical method, Bisection method, Regula-falsi method, Secant method, Iteration method, NewtonRaphson method, Bairstow's method and Graeffe's root squaring method and Newton's-iterative method, etc. Several formulae of the numerical method is described in the books of S.S. Sastry [3], R.L. Burden [1], A.R. Vasishtha [2] as well as there are lots of paper involving nonlinear Algebraic, Transcendental equations like improvement in Newton-Rapson method for nonlinear equations applying modified Adomian Decomposition method [4], on modified Newton method for solving a nonlinear algebraic equations by mid-point [10], New three-steps iterative method for solving nonlinear equations [7], A New two-step method for solving nonlinear equations [9], as well as a new method for solving nonlinear equations by Taylor expansion [8]. Besides, some investigations $[5,6,16]$ have shown a better way to interpret the algebraic and transcendental equation's root whereas Mohammad Hani Almomani et al. [11] presents a method for selecting the best performance systems. What is more, in [12], Zolt 'an Kov'acs et al. analyzed in understanding the convergence and stability of the Newton-Raphson method. In [13], Fernando Brambila Paz et al. calculated and demonstrated the fractional Newton-Raphson method accelerated with Aitken's method. Robin Kumar et al. [14] examined five numerical methods for their convergence. In [15] Nancy Velasco et al. implemented a Graphical User Interface in Matlab to find the roots of simple equations and more complex expressions. However, Kamoh Nathaniel Mahwash et al. [16] represents the two basic methods of approximating the solutions of nonlinear systems of algebraic equations. In [17], Farooq Ahmed Shah et al. suggest and analyze a new recurrence relation which creates the iterative methods of higher order for solving nonlinear equation $\mathrm{f}(\mathrm{x})=0$. Also, M. A. Hafiz et al. [18] propound and analyse a new predictor-corrector method for solving a nonlinear system of equations using the weight combination of mid-point, Trapezoidal and quadrature formulas.

In our working procedure, we have discussed and also compared with the existing some iterative methods, for example, Newton's method, False Position method, Secant method as well as Bisection method to achieve the best method among them. This research paper based on analyzing the best method to impart the best concept for solving the nonlinear equations. With the help of this paper, we can easily perceive how to determine the approximate roots of non-linear equations speedily. Moreover, we demonstrated some function to obtain an effective method which gives a smaller level of iteration among the mentioned methods.

\section{SCOPES AND OBJECTIVES OF THE STUDY}

The main scopes of the study are to evaluate the best method for solving nonlinear equations applying numerical methods. The objectives are given below;

Finding the rate of convergence, proper solution, as well as the level of errors of the methods.

$>$ Comparing the existed methods to achieve the best method for solving nonlinear equations.

\section{Materials ANd Methods}

Due to these works, the following methods is discussed such as Newton-Raphson method, False Position method, Secant method and Bisection method.

\subsection{Newton-Raphson Method}

Newton-Raphson method is the famous numerical method that is almost applied to solve the 
Applied Mathematics and Sciences: An International Journal (MathSJ), Vol. 6, No. 2/3, September 2019 equation's root and it helps to evaluate the roots of an equation $f(x)=0$ where $f(x)$ is considered to have a derivative of $\mathrm{f}^{\prime}(\mathrm{x})$. The basic idea of Newton-Raphson

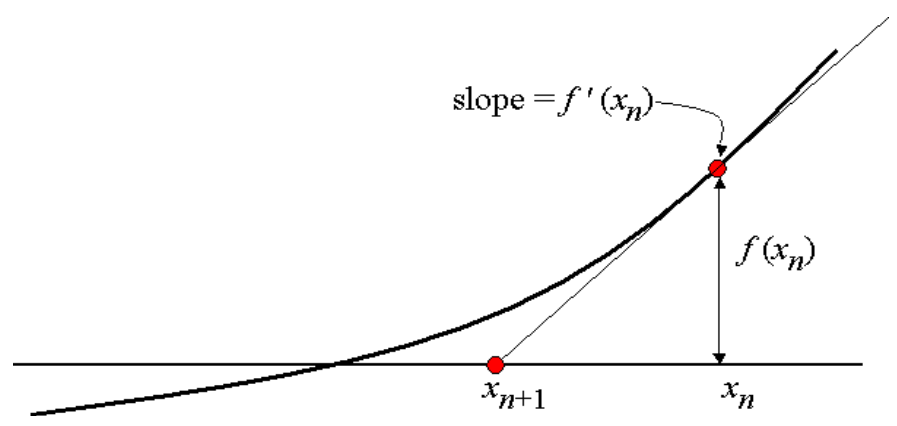

Figure 1. Newton-Raphson method

Also, Newton method can be achieved from the Taylor's series expansion of the function $\mathrm{f}(\mathrm{x})$ at the point $x 0$ as follows:

$$
f(x)=f\left(x_{0}\right)+\left(x-x_{0}\right) f^{\prime}\left(x_{0}\right)+\frac{\left(x-x_{0}\right)^{2}}{2 !} f^{\prime \prime}\left(x_{0}\right)+\cdots
$$

Since $(x-x 0)$ is small, we can neglect second, third and higher degree terms in $(x-x 0)$ and thus we obtain

$$
(x)=f(x 0)+(x-x 0) f^{\prime}(x 0)
$$

Putting $f(x)=0$ in $(1)$ we get,

Hence,

$$
\begin{gathered}
f\left(x_{0}\right)+\left(x-x_{0}\right) f^{\prime}\left(x_{0}\right)=0 \\
\text { Or, }\left(x-x_{0}\right)=-\frac{f\left(x_{0}\right)}{f^{\prime}\left(x_{0}\right)} ; f^{\prime}\left(x_{0}\right) \neq 0 \\
x=x_{0}-\frac{f\left(x_{0}\right)}{f^{\prime}\left(x_{0}\right)}
\end{gathered}
$$

Generalizing, we get the general formula

$$
x_{n+1}=x_{n}-\frac{f\left(x_{n}\right)}{f^{\prime}\left(x_{n}\right)} \text { for } n=0,1,2, \ldots
$$

Which is called Newton-Raphson formula.

Example: Solve the equation $\mathrm{f}(\mathrm{x})=x 3+2 x 2+10 x-20=0$ using Newton's method.

Given that, $\mathrm{f}(\mathrm{x})=x_{3}+2 x_{2}+10 x-20=0$

$\therefore f^{\prime}(x)=3 x 2+4 x+10$

Apply Newton's method to the equation, correct to five decimal places.

Now we can see that $\mathrm{f}(1)=-7<0$ and $\mathrm{f}(2)=16>0$.

Therefore the root lies between 1 and 2 .

We know that, Newton's formula as follows, 
Applied Mathematics and Sciences: An International Journal (MathSJ), Vol. 6, No. 2/3, September 2019

Or,

$$
\begin{aligned}
& x_{n+1}=x_{n}-\frac{f\left(x_{n}\right)}{f^{\prime}\left(x_{n}\right)} \\
& x_{n+1}=x_{n}-\left[\frac{x_{n}{ }^{3}+2 x_{n}{ }^{2}+10 x_{n}-20}{3 x_{n}{ }^{2}+4 x_{n}-10}\right]
\end{aligned}
$$

Solving the right hand side yields

$$
x_{n+1}=\frac{2\left(x_{n}^{3}+2 x_{n}^{2}+10\right)}{3 x_{n}^{2}+4 x_{n}-10}
$$

Convergence of Newton-Raphson method:

Newton's method is given below,

$$
x_{n+1}=x_{n}-\frac{f\left(x_{n}\right)}{f^{\prime}\left(x_{n}\right)}
$$

This is really an iteration method where

$$
x_{n+1}=\emptyset\left(x_{n}\right) ; \emptyset\left(x_{n}\right)=x_{n}-\frac{f\left(x_{n}\right)}{f^{\prime}\left(x_{n}\right)}
$$

In general, $\mathrm{x}=\emptyset(x)$, where $\emptyset(x)=x-\frac{f\left(x_{n}\right)}{f^{\prime}\left(x_{n}\right)}$.

We know that the iteration method converges if

$$
\left|\emptyset^{\prime}(x)\right|<1 \text {, i.e }\left|1-\frac{\left[f^{\prime}(x)\right]^{2}-f(x) f^{\prime \prime}(x)}{\left[f^{\prime}(x)\right]^{2}}\right|<1
$$

Or, $\quad\left|\frac{f(x) f^{\prime \prime}(x)}{\left[f^{\prime}(x)\right]^{2}}\right|<1$

$$
\text { i.e } \quad\left|\mathrm{f}(\mathrm{x}) \mathrm{f}^{\prime \prime}(\mathrm{x})\right|<\left[f^{\prime}(x)\right]^{2}
$$

Therefore, Newton-Raphson formula is convergent if $\left|\mathrm{f}(\mathrm{x}) \mathrm{f}^{\prime \prime}(\mathrm{x})\right|<\left[f^{\prime}(x)\right]^{2}$.

\subsection{False Position method}

False position method, which is a very old algorithm for solving a root finding problem. It is called trial and error technique that exerts test values for the sake of variable and after that adjusting this test value in imitation of the outcome. This formula is also called as "guess and check". The basic concept of this method is demonstrated in the following figure,

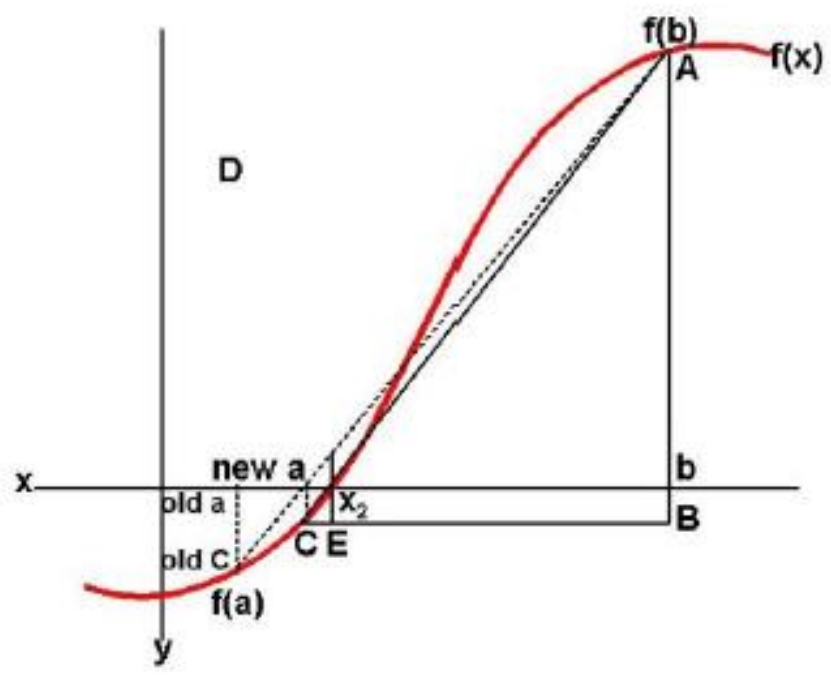


Figure 2. False Position method

Consider the equation $\mathrm{f}(\mathrm{x})=0$, where $\mathrm{f}(\mathrm{x})$ is continuous. In this method we choose two points a and $b$ such that $f(a)$ and $f(b)$ be of opposite signs $(a<b)$. So the curve of $y=f(x)$ will meet the $x-$ axis between $A(a, f(a))$ and $B(b, f(b))$. Hence a root lies between these two points. Now the equation of the chord joining the points $\mathrm{A}(\mathrm{a}, \mathrm{f}(\mathrm{a}))$ and $\mathrm{B}(\mathrm{b}, \mathrm{f}(\mathrm{b}))$ is given below

$$
\frac{x-a}{a-b}=\frac{y-f(a)}{f(a)-f(b)} .
$$

The point of intersection of the chord equation for the $\mathrm{x}$-axis imparts the first approximation $x 0$ for the root of $f(x)=0$, that is achieved by substituting $y=0$ in (1) we get,

$$
\begin{aligned}
& \frac{x_{0}-a}{a-b}=\frac{-f(a)}{f(a)-f(b)} \\
& \text { Or, } \quad x_{0}=a-\frac{f(a)(a-b)}{f(a)-f(b)} \\
& \therefore \quad x_{0}=\frac{a f(a)-b f(a)}{f(b)-f(a)} \ldots .
\end{aligned}
$$

If $\mathrm{f}(\mathrm{a})$ and $\mathrm{f}\left(x_{0}\right)$ be of opposite signs, so its root is between a and $x_{0}$. Then replacing b by $x_{0}$ in (2), we obtain the next approximation $x_{1}$. But if $\mathrm{f}(\mathrm{a})$ and $\mathrm{f}\left(x_{0}\right)$ are same sign, so $\mathrm{f}\left(x_{0}\right)$ and $\mathrm{f}(\mathrm{b})$ be of opposite signs, therefore its root is between $x_{0}$ and $\mathrm{b}$. Again, replacing a by $x_{0}$ in (2) we find $x 1$. This work is continued till the root is achieved its desired accuracy.The genearl formula is

$$
x_{n+1}=\frac{a f(a)-b f(a)}{f(b)-f(a)}
$$

Example: Solve the equation $\mathrm{f}(\mathrm{x})=x_{3}-3 x-5=0$ by the method of False Position method.

Solution:

$$
\begin{gathered}
\text { Let } \mathrm{f}(\mathrm{x})=x 3-3 x-5=0 \\
\text { Since } \mathrm{f}(2)=-3<0 \\
\mathrm{~F}(3)=13>0
\end{gathered}
$$

We know that,

$$
x_{0}=\frac{a f(a)-b f(a)}{f(b)-f(a)}
$$

When $\mathrm{a}=2, \mathrm{~b}=3$ then

$$
x_{0}=\frac{a f(a)-b f(a)}{f(b)-f(a)}=\frac{2 \times 13-3 \times-3}{13+3}=2.1875
$$

Now $f\left(x_{0}\right)=f(2.1875)=-1.095<0$.

The root lies between 2.1875 and 3 such that $\mathrm{a}=2.1875$ and $\mathrm{b}=3$ etc

Convergence of False Position method:

We know that,

$$
x_{n+1}=x_{n}-\left[\frac{\left(x_{n}-x_{n-1}\right)}{f\left(x_{n}\right)-f\left(x_{n-1}\right)}\right] f\left(x_{n}\right)
$$


Applied Mathematics and Sciences: An International Journal (MathSJ), Vol. 6, No. 2/3, September 2019

In the equation $\mathrm{f}(\mathrm{x})=0$, if the function $\mathrm{f}(\mathrm{x})$ is convex in the interval $\left(x_{0}, x_{1}\right)$ that takes a root then one of the points $x_{0}$, or $x_{1}$ is always fixed and the other point varies with $\mathrm{n}$. If the point $x_{0}$ is fixed , then the function $\mathrm{f}(\mathrm{x})$ is approximated by the straight line passing through the points $\left(x_{0}, f 0\right)$ as well as $\left(x_{n}, n\right), \mathrm{n}=1,2, \ldots \ldots$

Now, the error equation (2) from the convergence of secant method becomes

$$
\varepsilon_{n+1}=C \varepsilon_{n} \varepsilon_{n-1} \ldots \ldots(1)
$$

From equation (1), we get

$$
\begin{aligned}
& \varepsilon_{n+1}=C \varepsilon_{n} \varepsilon_{0} \\
& \varepsilon_{n+1}=C \varepsilon_{n} \varepsilon_{0}
\end{aligned}
$$

Where $\mathrm{C}=\frac{1}{2} \frac{f^{\prime \prime}(\zeta)}{f^{\prime}(\zeta)}$ and $\varepsilon_{0}=x_{0}-\zeta$ is independent of $\mathrm{k}$. Therefore we can write

$$
\varepsilon_{n+1}=C^{\prime} \varepsilon_{n}
$$

Where $\mathrm{C}=\mathrm{C} \varepsilon 0$ is the asymptotic error constant. Hence, the false Position method has Linear rate of Convergence.

\subsection{Secant Method}

The secant method is just a variation from the Newton's method. The basic idea is shown below,

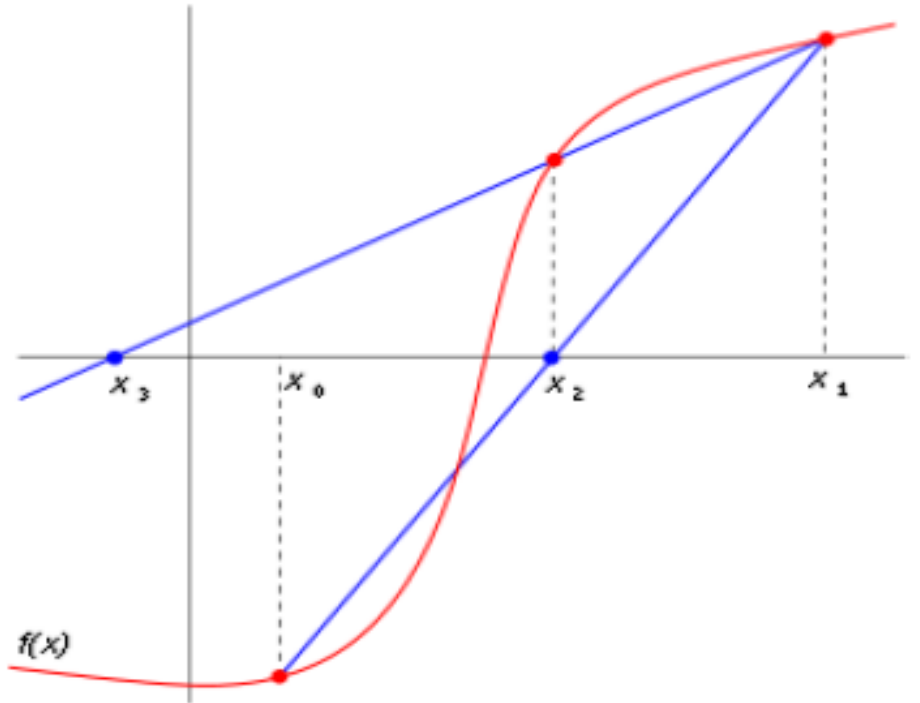

Figure 3. Secant method

The Newton method of solving a nonlinear equation $\mathrm{f}(\mathrm{x})=0$ is given by the iterative formula.

$$
x_{n+1}=x_{n}-\frac{f\left(x_{n}\right)}{f^{\prime}\left(x_{n}\right)} \ldots \ldots \ldots \ldots
$$

One of the drawbacks of the Newton's method is that one has to evaluation the derivative of the function. To overcome these drawbacks, the derivative of the function $\mathrm{f}(\mathrm{x})$ is approximated as

$$
f^{\prime}\left(x_{n}\right)=\frac{f\left(x_{n}\right)-f\left(x_{n-1}\right)}{x_{n}-x_{n-1}} \ldots \ldots \ldots
$$

Putting equation (2) into (1), we get, 
Applied Mathematics and Sciences: An International Journal (MathSJ), Vol. 6, No. 2/3, September 2019

By factoring out $\mathrm{f}\left(x_{n}\right)$

$$
x_{n+1}=x_{n}-\frac{x_{n} f\left(x_{n}\right)-x_{n-1} f\left(x_{n}\right)}{f\left(x_{n}\right)-f\left(x_{n-1}\right)}
$$

This can also be written as

$$
x_{n+1}=x_{n}-\frac{f\left(x_{n}\right)\left(x_{n}-x_{n-1}\right)}{f\left(x_{n}\right)-f\left(x_{n-1}\right)}
$$

$$
x_{n+1}=x_{n}-\left[\frac{\left(x_{n}-x_{n-1}\right)}{f\left(x_{n}\right)-f\left(x_{n-1}\right)}\right] f\left(x_{n}\right)
$$

The above equation is called the secant method. This is like Newton-Raphson method but requires two initial guess.

Example: Solve the equation $\mathrm{f}(\mathrm{x})=x_{3}-2 x-5=0$ using secant method.

Let the two initial approximations be given by

$$
x-1=2 \text { and } x 0=3
$$

The root lies between 2 and 3 .

By secant method, first approximation is when $\mathrm{i}=1 ; x_{2}=x_{1}-\frac{f\left(x_{1}\right)\left(x_{1}-x_{0}\right)}{f\left(x_{1}\right)-f\left(x_{0}\right)}$

$$
\begin{array}{ll}
\text { when } \mathrm{i}=2 ; & x_{3}=x_{2}-\frac{f\left(x_{2}\right)\left(x_{2}-x_{1}\right)}{f\left(x_{2}\right)-f\left(x_{1}\right)} \\
\text { when } \mathrm{i}=3 ; & x_{4}=x_{3}-\frac{f\left(x_{3}\right)\left(x_{3}-x_{2}\right)}{f\left(x_{3}\right)-f\left(x_{2}\right)} \\
\text { when } \mathrm{i}=4 ; & x_{5}=x_{4}-\frac{f\left(x_{4}\right)\left(x_{4}-x_{3}\right)}{f\left(x_{4}\right)-f\left(x_{3}\right)}
\end{array}
$$

Convergence of Secant method:

We know that,

$$
x_{n+1}=x_{n}-\left[\frac{\left(x_{n}-x_{n-1}\right)}{f\left(x_{n}\right)-f\left(x_{n-1}\right)}\right] f\left(x_{n}\right) .
$$

We assume that $\zeta$ is a simple root of $\mathrm{f}(\mathrm{x})=0$. Putting $x_{n}=\zeta+\epsilon_{k}$ in (1) we obtain

$$
\epsilon_{n+1}=\epsilon_{n}-\left[\frac{\left(\epsilon_{n}-\epsilon_{n-1}\right)}{f\left(\zeta+\epsilon_{n}\right)-f\left(\zeta+\epsilon_{n-1}\right)}\right] f\left(\zeta+\epsilon_{n}\right)
$$

Expanding $\mathrm{f}\left(\zeta+\epsilon_{k}\right)$ and $\mathrm{f}\left(\zeta+\epsilon_{k-1}\right)$ in taylor's series about the point $\zeta$ and noting that $\mathrm{f}(\zeta)=0$ we get,

$$
\varepsilon_{n+1}=\epsilon_{n}-\frac{\left(\epsilon_{n}-\epsilon_{n-1}\right)\left[\epsilon_{n} f^{\prime}(\zeta)+\frac{1}{2} \epsilon_{n}^{2} f^{\prime \prime}(\zeta)+\cdots\right]}{\left(\epsilon_{n}-\epsilon_{n-1}\right) f^{\prime}(\zeta)+\frac{1}{2}\left(\epsilon_{n}^{2}-\epsilon_{n-1}^{2}\right) f^{\prime \prime}(\zeta)+\cdots}
$$

Or, $\varepsilon_{n+1}=\epsilon_{n}-\left[\epsilon_{n} f^{\prime}(\zeta)+\frac{1}{2} \epsilon_{n}^{2} f^{\prime \prime}(\zeta)+\cdots\right]\left[1+\frac{1}{2}\left(\epsilon_{k-1}+\varepsilon_{k}\right) f^{\prime \prime}(\zeta) / f^{\prime}(\zeta)+\cdots\right]^{-1}$

Or, $\varepsilon_{n+1}=\frac{1}{2} \varepsilon_{n} \varepsilon_{n-1} f^{\prime \prime}(\zeta) / f^{\prime}(\zeta)+0\left(\varepsilon_{n}^{2} \varepsilon_{n-1}+\varepsilon_{n}^{2} \varepsilon_{n-1}\right)$

Or, $\varepsilon_{n+1}=C \varepsilon_{n} \varepsilon_{n-1}$

Where $\mathrm{C}=1 / 2 f^{\prime \prime}(\zeta) / f^{\prime}(\zeta)$ and the higher powers of $\varepsilon n$ are neglected. 
Where $\mathrm{C}=1 / 2 f^{\prime \prime}(\zeta) / f^{\prime}(\zeta)$ and the higher powers of $\varepsilon_{n}$ are neglected.

The relation of the form (2) is called the error equation. Keeping in mind the definition of convergence we want a relation of the form, $\varepsilon_{n+1}=A \varepsilon_{k}^{p}$.

Where $\mathrm{A}$ and $\mathrm{p}$ are to be determined.

From (3) we have $\varepsilon_{n}=A \varepsilon_{n-1}^{p}$ or $\varepsilon_{n+1}=A^{-1 / p} \varepsilon_{n}^{1 / p}$

Putting the value of $\varepsilon_{n+1}$ and $\varepsilon_{n-1}$ in (1)

Comparing the power of $\varepsilon_{n}$ on both sides we get

$$
\varepsilon_{n}^{p}=C A^{-\left(1+\frac{1}{p}\right)} \varepsilon_{n}^{1+1 / p}
$$

$\mathrm{p}=1+1 / \mathrm{p}$ which gives $\mathrm{p}=1 / 2(1 \pm \sqrt{5})$.

Neglecting the negative sign, we get the rate of convergence for the Secant method is $p=1.618$.

\subsection{Bisection Method}

The bisection method is very facile and perhaps always faithful to trace a root in numerical schemes for solving non-linear equations. It is frequently applied to acquire of searching a root value of a function and based on the intermediate value theorem (IVT). This method always succeeds due to it is an easier way to perceive. The basic concept of this method is given below;

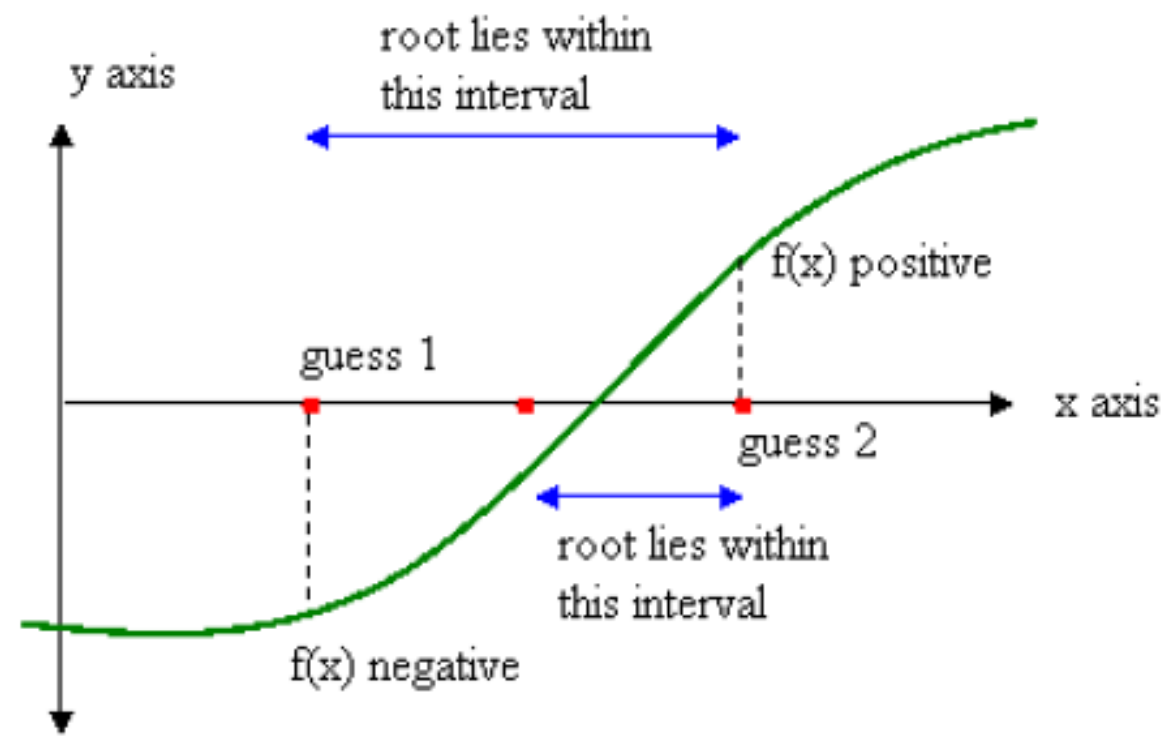

Figure 4. Bisection method

This Bisection method states that if $f(x)$ is continuous which is defined on the interval $[a, b]$ satisfying the relation $f(a) f(b)<0$ with $f(a)$ and (b) of opposite signs then by the intermediate value theorem(IVT), it has at least a root or zero of $\mathrm{f}(\mathrm{x})=0$ within the interval [a, $\mathrm{b}]$. It consists of finding two real numbers $a$ and $b$, having the interval $[a, b]$, as well as the root, lies in $a \leq x \leq b$ whose length is, at each step, half of its original interval length. This procedure is repeated until the interval imparts required accuracy by virtue of the requisite root is achieved. While it has more than one root on the interval $(a, b)$, as a result, the root must be unique. Moreover, the root converges linearly and very slowly. But when $\mathrm{f}(x 0)$ is very much nearly zero or is very small then we can stop the iteration. Therefore, the general formula is

$$
x_{n}=\frac{a+b}{2}
$$


Applied Mathematics and Sciences: An International Journal (MathSJ), Vol. 6, No. 2/3, September 2019

Example: Solving the equation $\mathrm{f}(\mathrm{x})=x^{3}-x-1=0$ by bisection method correct up to two decimal places.

Solution: Let $\mathrm{f}(\mathrm{x})=x^{3}-x-1$

$$
\begin{aligned}
& \therefore \mathrm{f}(1)=-1<0 \\
& \text { And } \mathrm{f}(2)=5>0
\end{aligned}
$$

So at least one root of the given equation lies between 1 and 2 .

$$
\begin{aligned}
& \text { Let } x_{0}=\frac{a+b}{2}=\frac{1+2}{2}=1.5 . \\
& \mathrm{f}\left(x_{0}\right)=0.875>0
\end{aligned}
$$

The root lies between 1 and 1.5 etc.

Convergence of Bisection method:

Let us conventionally define the "approximation" at $x_{n}$ after the n-th iteration as the midpoint

$$
x_{n}=\frac{a_{n}+b_{n}}{2}
$$

$$
\begin{array}{r}
\text { of } \mathrm{I}_{n} \text {. Since the actual solution } \mathrm{f}(\mathrm{c})=0 \text { satisfies } \mathrm{c} \in \mathrm{I}_{n} \text {, we have } \\
\qquad\left|x_{n}-\mathrm{c}\right| \leq 1 / 2\left|\mathrm{I}_{n}\right|
\end{array}
$$

where $\left|\mathrm{I}_{n}\right|$ symbolizes the length of the interval $\mathrm{I}_{n}$. Since the length of the current search interval gets divided in half in each iteration, we have,

$$
\left|e_{n}\right|=\left|x_{n}-\mathrm{c}\right| \leq\left(\frac{1}{2}\right)^{n}\left|\mathrm{I}_{0}\right|
$$

We interpret this behavior as linear convergence; although we cannot strictly guarantee that $\left|e_{n+1}\right| \leq$ $L\left|e_{n}\right|(L<1)$ at each iteration, then the convergence is called the first order and $\mathrm{L}$ is called the rate of convergence.

\subsection{Comparison Of The Rate Of Convergence Among The Four Method}

The rate of convergence is a very essential issue for the sake of solution of algebraic and transcendental equations, due to the rate of convergence of any numerical method evaluates the speed of the approximation to the solution of the problems.

Following table demonstrates the comparison of rate of convergence.

Table 1. Comparison of the rate of convergence

\begin{tabular}{|c|c|c|}
\hline Method & Based on Equation & Rate of Convergence \\
\hline Newton-Raphson & $1^{\text {st }}$ degree & 2 \\
\hline False Position & $1^{\text {st }}$ degree & 1 \\
\hline Secant & $1^{\text {st }}$ degree & 1.618 \\
\hline Bisection & $1^{\text {st }}$ degree & 0.5 \\
\hline
\end{tabular}

From this table, we see that Newton-Raphson has a better rate of convergence than other methods i.e. 2 . 


\section{ANALYSIS AND COMPARISON}

\subsection{Graphical Development Of The Approximation Root To Achieve The Best Method}

The graphical development of the four method is illustrated as follows,
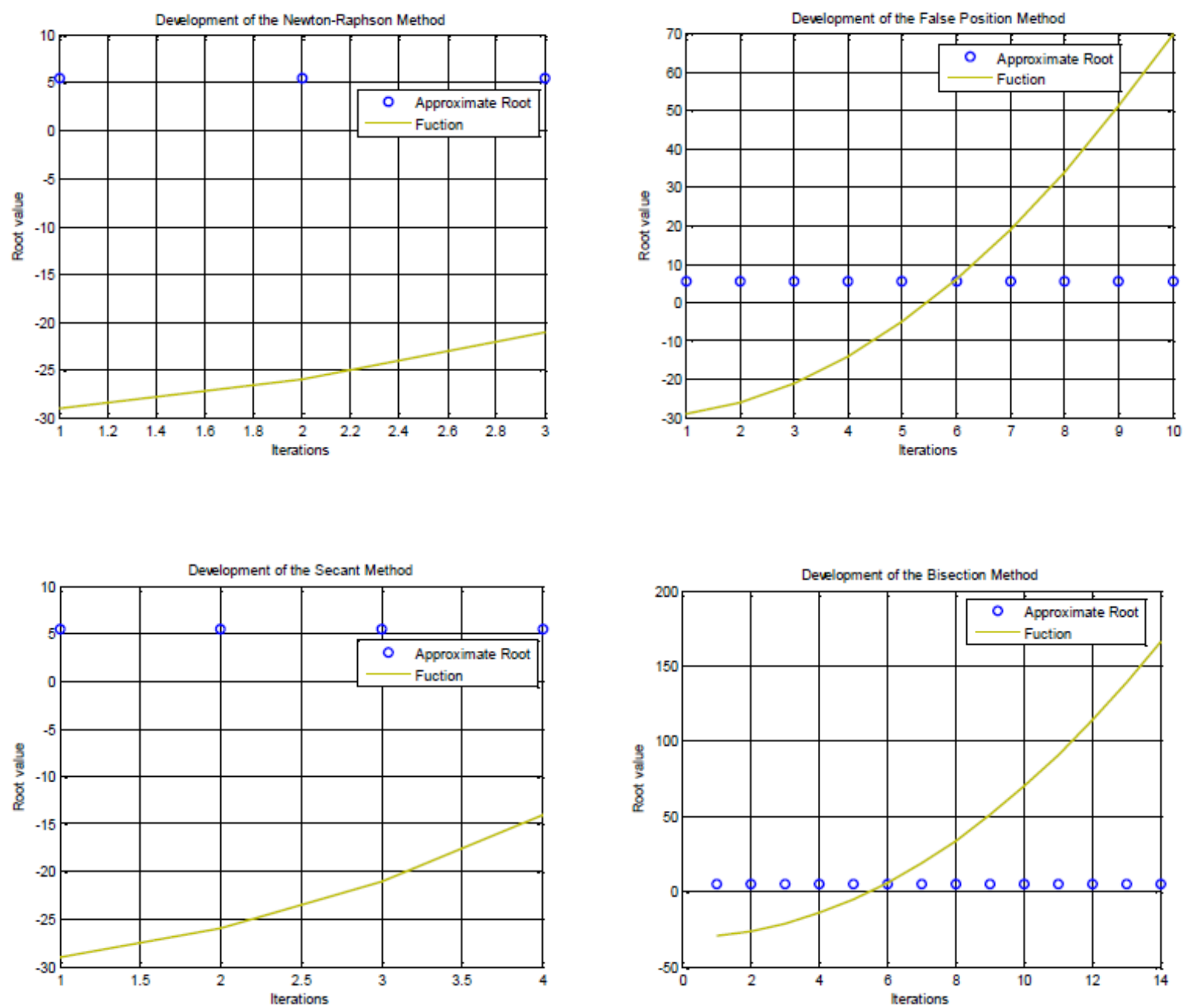

Figure 5. Graphical development among the four methods. The function used is $x 2-30$ with the interval [5 $6]$ and the desired error of 0.0001 .

From the above graph, for the Newton-Raphson method, in each iteration, the graph demonstrates the approximate root with only 3 iterations as it converges more rapidly and accurately whereas other method delays to give desire roots of the equation. In the Bisection method, it requires a large number of iterations to meet the desired root as convergent slowly. For the Secant method, it imparts a level of convergence but not as compared to the Newton method. The False Position method converges more slowly due to it needs at least 10 iterations to compare to the Newton and secant method respectively.

Both algorithms are tested with several functions taking as data for error 0.0001 and the desired accuracy. The results gained for the approximate root, error, and iteration are summarized in table 2.6.1 for the Newton-Raphson method, in table 2.6.2 for False-Position method, in table 2.6.3 for 
Applied Mathematics and Sciences: An International Journal (MathSJ), Vol. 6, No. 2/3, September 2019

Secant method and in table 2.6.4 for Bisection method. The Newton-Raphson imparts the small number of iteration correctly as long as the initial values enclose the desired root.

Table 2. Data obtained with the Newton-Raphson Method

\begin{tabular}{|c|c|c|c|}
\hline Function Type & Approximate Root & Error & Iteration \\
\hline Linear: $3 \mathrm{x}+5$ & -1.6667 & 0.0000000 & 2 \\
\hline Quadratic: $x^{2}-12$ & 3.46410 & $4.8917 \mathrm{e}-09$ & 4 \\
\hline Cubic: $x^{3}-48$ & 3.6342 & $7.9622 \mathrm{e}-06$ & 4 \\
\hline Trigonometric: $3 x-\cos (\mathrm{x})-1$ & 0.60710 & $1.7597 \mathrm{e}-08$ & 3 \\
\hline Exponential: $\mathrm{x} e^{x}-2$ & 0.85261 & $1.2179 \mathrm{e}-07$ & 3 \\
\hline Logarithmic: $x^{2}+\ln x-2$ & 1.31410 & $7.7547 \mathrm{e}-05$ & 3 \\
\hline Combination: $\cos (x)-\mathrm{x} e^{x}$ & 0.51776 & $1.9482 \mathrm{e}-08$ & 5 \\
\hline Combination: $3 x-\sqrt{1+\sin (x)}$ & 0.39185 & $3.7502 \mathrm{e}-06$ & 3 \\
\hline
\end{tabular}

In the False position method, it imparts a lot of iteration number to meet the desired root.

Table 3. Data obtained with the False Position Method

\begin{tabular}{|c|c|c|c|}
\hline Function Type & Approximate Root & Error & Iteration \\
\hline Linear: $4 \mathrm{x}+7$ & -1.66666 & 0.000000000000000 & 3 \\
\hline Quadratic: $x^{2}-12$ & 3.464101 & 0.000000000001810 & 10 \\
\hline Cubic: $x^{3}-48$ & 3.634241 & 0.000000000000003 & 14 \\
\hline Trigonometric: $3 x-\cos (\mathrm{x})-1$ & 0.607101 & 0.000000000000156 & 9 \\
\hline Exponential: $\mathrm{x} e^{x}-2$ & 0.85260 & 0.000000000002899 & 12 \\
\hline Logarithmic: $x^{2}+\ln x-2$ & 1.314096 & 0.000000000000001 & 17 \\
\hline Combination: $\cos (x)-\mathrm{x} e^{x}$ & 0.517757 & 0.000000000000061 & 15 \\
\hline Combination: $3 x-\sqrt{1+\sin (x)}$ & 0.391846 & 0.000000000000877 & 8 \\
\hline
\end{tabular}

We apply the secant method to obtain the result with a few numbers of iterations.

Table 4. Data obtained with the Secant Method

\begin{tabular}{|c|c|c|c|}
\hline Function Type & Approximate Root & Error & Iteration \\
\hline Linear: $4 \mathrm{x}+7$ & -1.66666 & 0.0000000 & 2 \\
\hline Quadratic: $x^{2}-12$ & 3.464101 & $1.3222 \mathrm{e}-05$ & 4 \\
\hline Cubic: $x^{3}-48$ & 3.634241 & $2.0674 \mathrm{e}-07$ & 5 \\
\hline Trigonometric: $3 x-\cos (\mathrm{x})-1$ & 0.6071016 & $3.8551 \mathrm{e}-06$ & 4 \\
\hline Exponential: $\mathrm{x} e^{x}-2$ & 0.8526054 & $1.2398 \mathrm{e}-05$ & 5 \\
\hline Logarithmic: $x^{2}+\ln x-2$ & 1.3140967 & $5.4035 \mathrm{e}-05$ & 4 \\
\hline Combination: $\cos (x)-\mathrm{x} e^{x}$ & 0.51776 & $9.9055 \mathrm{e}-06$ & 6 \\
\hline Combination: $3 x-\sqrt{1+\sin (x)}$ & 0.39185 & $3.5903 \mathrm{e}-07$ & 4 \\
\hline
\end{tabular}

Similarly, the Bisection method demonstrates the roots slowly as long as the initial values enclose the desired root as well as gives a large number of iteration. 
Applied Mathematics and Sciences: An International Journal (MathSJ), Vol. 6, No. 2/3, September 2019

Table 5. Data obtained with the Bisection Method

\begin{tabular}{|c|c|c|c|}
\hline Function Type & Approximate Root & Error & Iteration \\
\hline Linear: $4 \mathrm{x}+7$ & -1.66664 & 0.000076293 & 16 \\
\hline Quadratic: $x^{2}-12$ & 3.464050 & 0.000355567 & 14 \\
\hline Cubic: $x^{3}-48$ & 3.634241 & 0.000223494 & 15 \\
\hline Trigonometric: $3 x-\cos (\mathrm{x})-1$ & 0.607102 & 0.000164184 & 13 \\
\hline Exponential: $\mathrm{x} e^{x}-2$ & 0.852606 & 0.000109137 & 15 \\
\hline Logarithmic: $x^{2}+\ln x-2$ & 1.314097 & 0.001207722 & 9 \\
\hline Combination: $\cos (x)-\mathrm{x} e^{x}$ & 0.517761 & 0.001956944 & 10 \\
\hline Combination: $3 x-\sqrt{1+\sin (x)}$ & 0.391847 & 0.000076418 & 14 \\
\hline
\end{tabular}

\subsection{Comparison Of The Approximate Error}

A comparison of the approximate error to find the root of the function $x^{4}-x-10$ with four methods within the interval [1 2 ] of an accuracy 0.0001 is demonstrated in the following figure 3.2 .

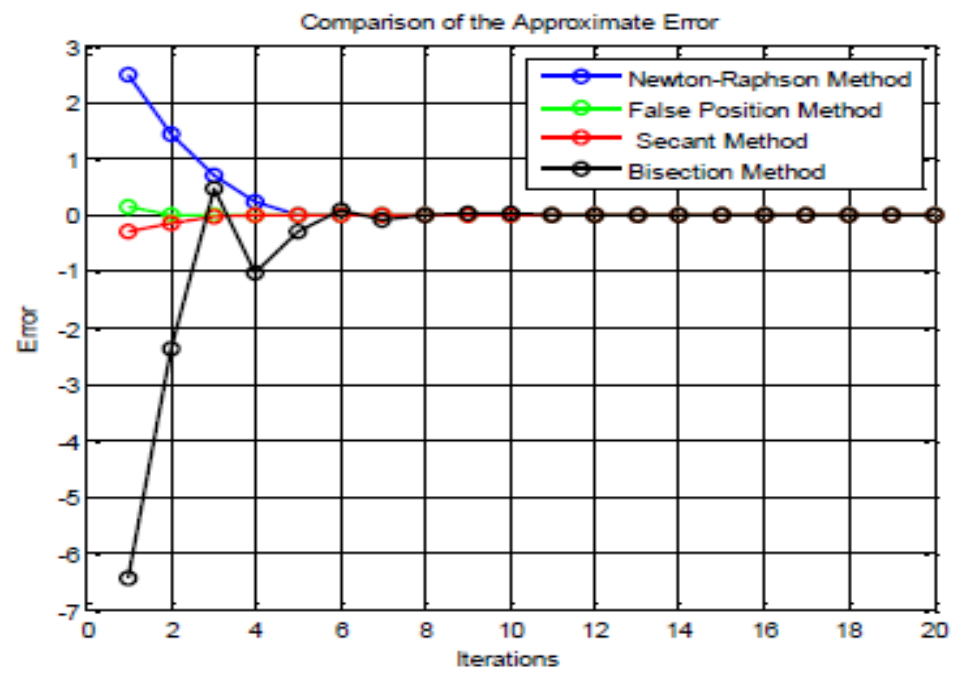

Figure 6. The approximate error is plotted against the number of iterations (1-20).

From the above graphical development and comparison, it is recommended that NewtonRaphson's method is the fastest in the rate of convergence compared with the other methods.

\section{Test And Results To Verify The Best Method}

Following examples interpret the result achieved by the four methods to solve nonlinear equations such as algebraic and transcendental equations.

Problem 1. Consider the following equation [2 3],

$$
f(x)=x^{3}-2 x-5=0
$$

We start $x_{0}=2$. 
Applied Mathematics and Sciences: An International Journal (MathSJ), Vol. 6, No. 2/3, September 2019

Table 7. Number of iterations and the results obtained by the four method

\begin{tabular}{|c|c|c|c|c|c|c|c|c|}
\hline $\mathrm{N}$ & $\begin{array}{c}\text { Newton- } \\
\text { Raphson } \\
\text { Method }\end{array}$ & Error & $\begin{array}{c}\text { False } \\
\text { Position } \\
\text { Method }\end{array}$ & Error & $\begin{array}{c}\text { Secant } \\
\text { Method }\end{array}$ & Error & $\begin{array}{c}\text { Bisection } \\
\text { Method }\end{array}$ & Error \\
\hline 1 & 2.12000 & 0.00544851 & 2.058823 & 0.03572795 & 2.05882 & 0.9411764 & 2.50000 & 5.6249937 \\
\hline 2 & 2.09513 & 0.00001663 & 2.081263 & 0.01328782 & 2.08126 & 0.0224401 & 2.25000 & 1.8906187 \\
\hline $\mathbf{3}$ & $\mathbf{2 . 0 9 4 5 5}$ & $\mathbf{1 . 5}$ & 2.089639 & 0.00491227 & 2.09482 & 0.0135604 & 2.12500 & 0.3456968 \\
\hline 4 & 2.09455 & 0.00000000 & 2.092739 & 0.00181190 & 2.09454 & 0.0002747 & 2.06250 & -0.351324 \\
\hline $\mathbf{5}$ & 2.09455 & 0.00000000 & 2.093883 & 0.00066777 & $\mathbf{2 . 0 9 4 5 5}$ & $\mathbf{0 . 0 0 0 0 0 2 0}$ & 2.09375 & -0.008947 \\
\hline 6 & 2.09455 & 0.00000000 & 2.094305 & 0.00024603 & 2.09455 & 0.0000000 & 2.10937 & 0.1668329 \\
\hline 7 & 2.09455 & 0.00000000 & 2.094460 & 0.00009063 & 2.09455 & 0.0000000 & 2.10156 & 0.0785560 \\
\hline 8 & 2.09455 & 0.00000000 & 2.094518 & 0.00003338 & 2.09455 & 0.0000000 & 2.09765 & 0.0347080 \\
\hline 9 & 2.09455 & 0.00000000 & 2.094539 & 0.00001229 & 2.09455 & 0.0000000 & 2.09570 & 0.0128560 \\
\hline 10 & 2.09455 & 0.00000000 & 2.094546 & 0.00000453 & 2.09455 & 0.0000000 & 2.09472 & 0.0019481 \\
\hline $\mathbf{1 1}$ & 2.09455 & 0.00000000 & $\mathbf{2 . 0 9 4 5 4 6}$ & $\mathbf{0 . 0 0 0 0 0 1 6 6}$ & 2.09455 & 0.0000000 & 2.09423 & -0.003501 \\
\hline 12 & 2.09455 & 0.00000000 & 2.094546 & 0.00000061 & 2.09455 & 0.0000000 & 2.09448 & -0.000777 \\
\hline 13 & 2.09455 & 0.00000000 & 2.094546 & 0.00000022 & 2.09455 & 0.0000000 & 2.09460 & 0.0005854 \\
\hline 14 & 2.09455 & 0.00000000 & 2.094546 & 0.00000008 & 2.09455 & 0.0000000 & 2.09454 & -0.000095 \\
\hline 15 & 2.09455 & 0.00000000 & 2.094546 & 0.00000003 & 2.09455 & 0.0000000 & 2.09455 & 0.0002448 \\
\hline $\mathbf{1 6}$ & 2.09455 & 0.00000000 & 2.094546 & 0.00000001 & 2.09455 & 0.0000000 & $\mathbf{2 . 0 9 4 5 5}$ & $\mathbf{0 . 0 0 0 0 7 4 5}$ \\
\hline 17 & 2.09455 & 0.00000000 & 2.094546 & 0.00000000 & 2.09455 & 0.0000000 & 2.09455 & -0.000010 \\
\hline 18 & 2.09455 & 0.00000000 & 2.094546 & 0.00000000 & 2.09455 & 0.0000000 & 2.09455 & 0.0000319 \\
\hline 19 & 2.09455 & 0.00000000 & 2.094546 & 0.00000000 & 2.09455 & 0.0000000 & 2.09455 & 0.0000106 \\
\hline 20 & 2.09455 & 0.00000000 & 2.094546 & 0.00000000 & 2.09455 & 0.0000000 & 2.09455 & 0.0000000 \\
\hline
\end{tabular}

Moreover, we can look at the following approximate error graph gained from the above four methods which illustrate the change of errors from one method to another method.

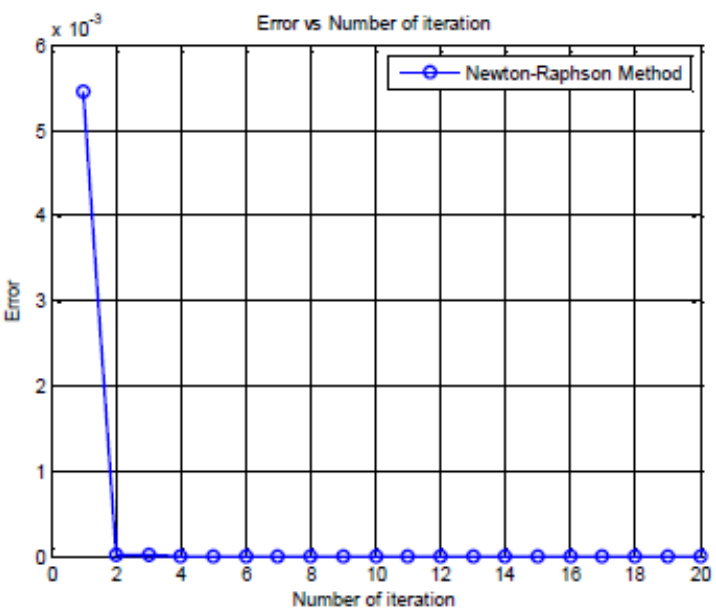

Figure 7. The graph of NRM

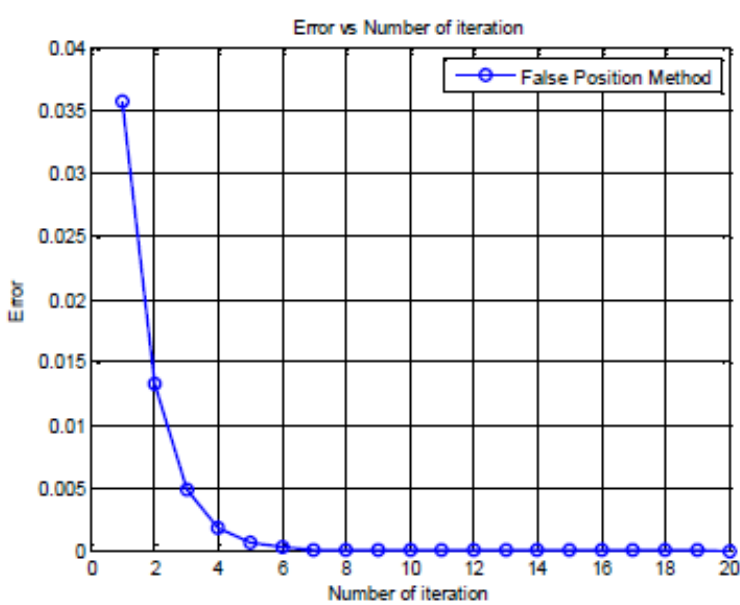

Figure 8. The graph of FPM 
Applied Mathematics and Sciences: An International Journal (MathSJ), Vol. 6, No. 2/3, September 2019

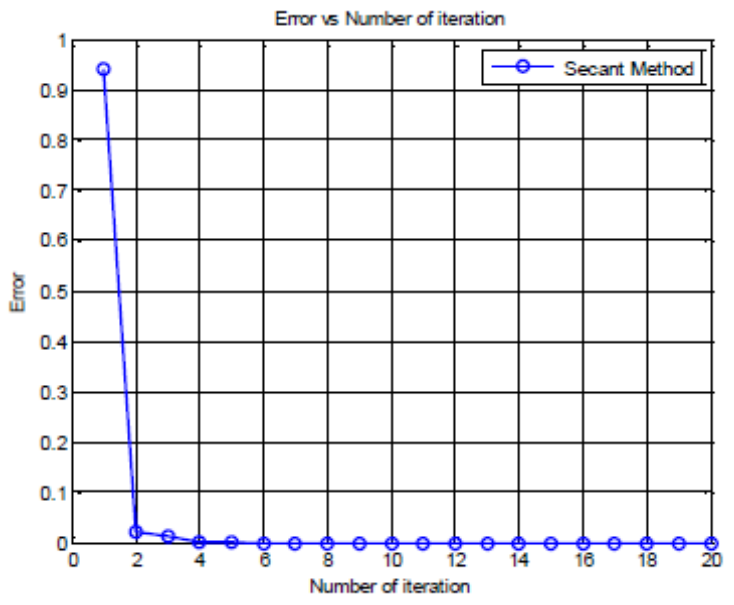

Figure 9. The graph of SM

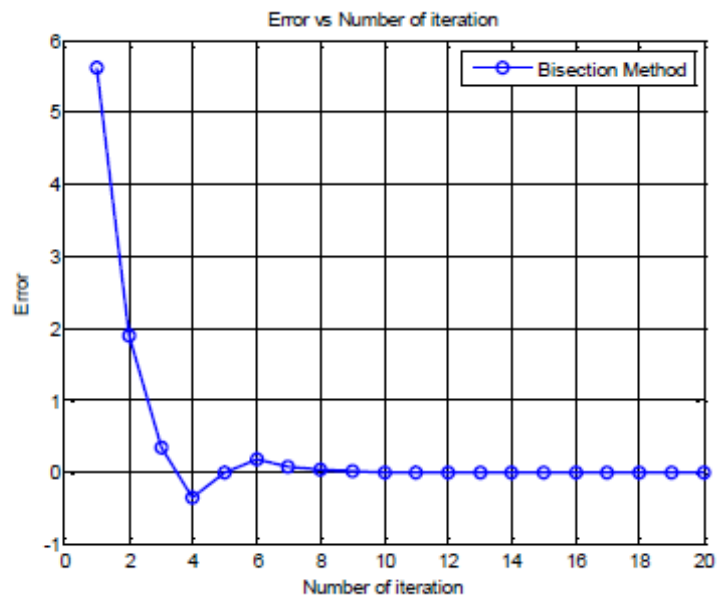

Figure 10. The graph of BM

Comparing the above graph, we see that Newton-Raphson Method is a better method than others.

Similarly,

Problem 2. Consider the following equation [-1 -2],

$$
f(x)=\sin x-1-x^{3}=0
$$

We start $x_{0}=-1$.

\begin{tabular}{|c|c|c|c|c|c|c|c|c|}
\hline $\mathrm{N}$ & $\begin{array}{l}\text { Newton- } \\
\text { Raphson } \\
\text { Method }\end{array}$ & Error & $\begin{array}{c}\text { False } \\
\text { Position } \\
\text { Method }\end{array}$ & Error & $\begin{array}{l}\text { Secant } \\
\text { Method }\end{array}$ & Error & $\begin{array}{l}\text { Bisection } \\
\text { Method }\end{array}$ & Error \\
\hline 1 & -1.27636 & -0.09305126 & -1.12138 & -0.1276658 & -1.12138 & 0.8786136 & -1.50000 & 1.377501 \\
\hline 2 & -1.24974 & -0.00738636 & -1.18687 & -0.0621759 & -1.18687 & 0.0654899 & -1.2500 & 0.004136 \\
\hline 3 & -1.24905 & -0.00005222 & -1.21958 & -0.0294663 & -1.25793 & 0.0710594 & -1.1250 & -0.47844 \\
\hline 4 & -1.24905 & $-2.6 \times 10^{-9}$ & -1.23527 & -0.0137771 & -1.24849 & 0.0094389 & -1.18750 & -0.25288 \\
\hline 5 & -1.24905 & 0.000000000 & -1.24265 & -0.0064000 & -1.24904 & 0.0005506 & -1.21875 & -0.12840 \\
\hline 6 & -1.24905 & 0.000000000 & -1.24608 & -0.0029641 & -1.24905 & 0.0000047 & -1.23437 & -0.06315 \\
\hline 7 & -1.24905 & 0.000000000 & -1.24768 & -0.0013708 & -1.24905 & 0.0000000 & -1.24218 & -0.02976 \\
\hline 8 & -1.24905 & 0.000000000 & -1.24841 & -0.0006335 & -1.24905 & 0.0000000 & -1.24609 & -0.01287 \\
\hline 9 & -1.24905 & 0.000000000 & -1.24875 & -0.0002926 & -1.24905 & 0.0000000 & -1.24804 & -0.00438 \\
\hline 10 & -1.24905 & 0.000000000 & -1.24891 & -0.0001351 & -1.24905 & 0.0000000 & -1.24902 & -0.00012 \\
\hline 11 & -1.24905 & 0.000000000 & -1.24891 & -0.0000624 & -1.24905 & 0.0000000 & -1.24951 & 0.002002 \\
\hline 12 & -1.24905 & 0.000000000 & -1.24891 & -0.0000287 & -1.24905 & 0.0000000 & -1.24926 & 0.000936 \\
\hline 13 & -1.24905 & 0.000000000 & -1.24891 & -0.0000132 & -1.24905 & 0.0000000 & -1.24914 & 0.000403 \\
\hline 14 & -1.24905 & 0.000000000 & -1.24891 & -0.0000060 & -1.24905 & 0.0000000 & -1.24908 & 0.000137 \\
\hline 15 & -1.24905 & 0.000000000 & -1.24891 & -0.0000027 & -1.24905 & 0.0000000 & -1.24907 & 0.000004 \\
\hline 16 & -1.24905 & 0.000000000 & -1.24891 & 0.0000000 & -1.24905 & 0.0000000 & -1.24907 & -0.00006 \\
\hline 17 & -1.24905 & 0.000000000 & -1.24891 & 0.0000000 & -1.24905 & 0.0000000 & -1.24907 & -0.00002 \\
\hline 18 & -1.24905 & 0.000000000 & -1.24891 & 0.0000000 & -1.24905 & 0.0000000 & -1.24907 & -0.00001 \\
\hline 19 & -1.24905 & 0.000000000 & -1.24891 & 0.0000000 & -1.24905 & 0.0000000 & -1.24907 & 0.000000 \\
\hline 20 & -1.24905 & 0.000000000 & -1.24891 & 0.0000000 & -1.24905 & 0.0000000 & -1.24907 & 0.000000 \\
\hline
\end{tabular}


Applied Mathematics and Sciences: An International Journal (MathSJ), Vol. 6, No. 2/3, September 2019

Table 8 . Number of iterations and the results obtained by the four method

Moreover, we can also look at the following approximate error graph gained from the above four methods which illustrate the change of errors from one method to another method.

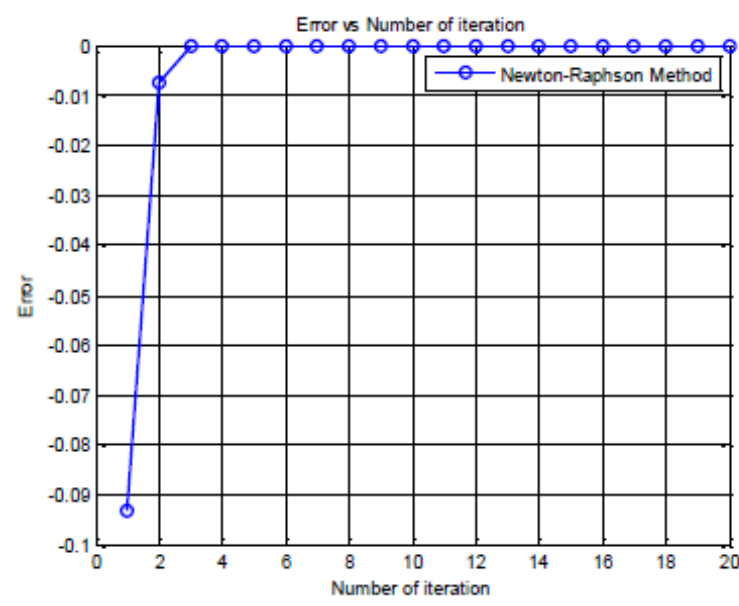

Figure 11. The graph of NRM

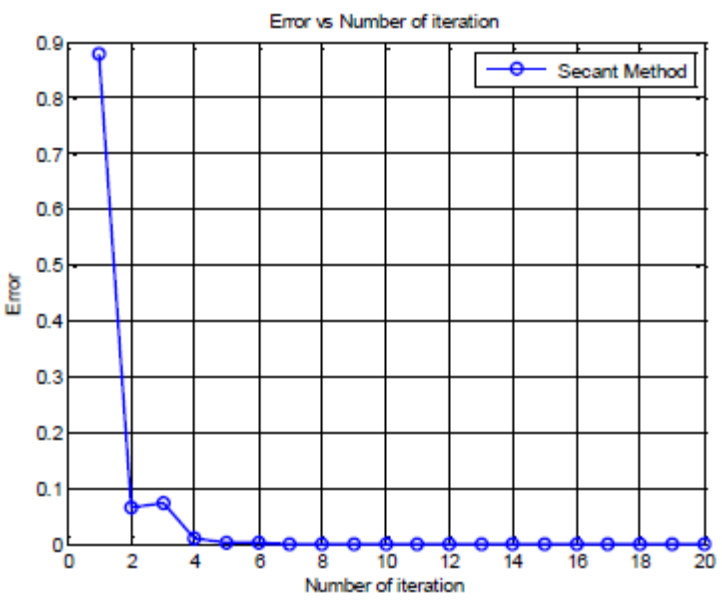

Figure 13. The graph of SM

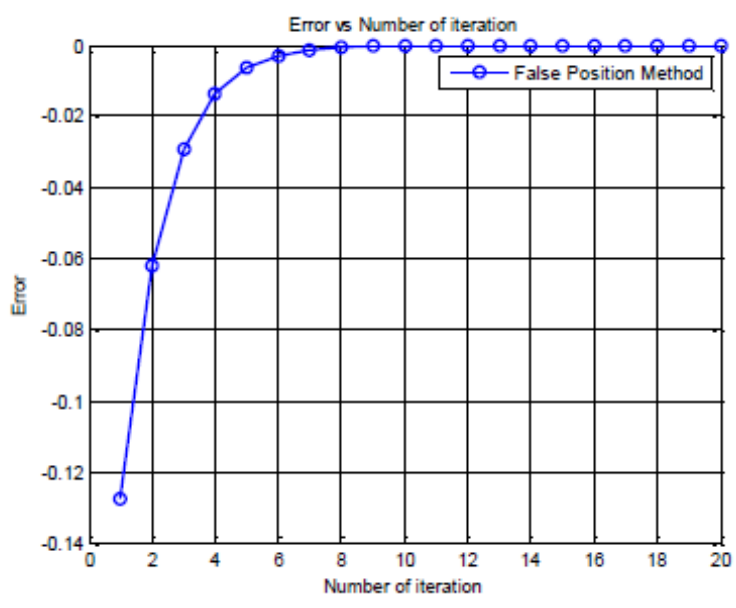

Figure 12. The graph of FPM

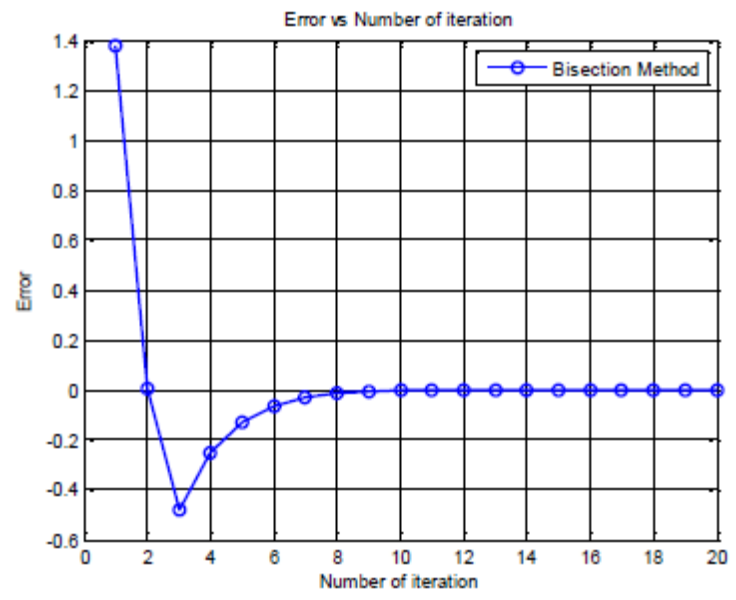

Figure 14. The graph of BM

Comparing the above graph, we see that Newton-Raphson Method is a better method than others.

\section{Conclusion}

In this research works, we have analyzed and compared the four iterative methods for solving non-linear equations that assist to present a better performing method. The MATLAB results are also delivered to check the appropriateness of the best method. With the help of the approximate error graph, we can say that the Newton's method is the most robust and capable method for the sake of solving the nonlinear equation. Besides, Newton's method gives a lesser number of iterations concerning the other methods and it shows less processing time. Ultimately, it is consulted steadily that Newton's method is the most effective and accurate owing to solving the nonlinear problems. 
Applied Mathematics and Sciences: An International Journal (MathSJ), Vol. 6, No. 2/3, September 2019

\section{REFERENCES}

[1] Richard L. Burden, J.douglas Faires, Annette M. burden, "Numerical Analysis", Tenth Edition, ISBN: 978-1-305-25366-7.

[2] R. Vasishtha, Vipin Vasishtha, "Numerical Analysis", Fourth Edition.

[3] S.S. Sastry, "Introduction Methods of Numerical Analysis", Fourth Edition, ISBN: 81-203-2761-6.

[4] Shin Min Kang, Waqas Nazeer, "Improvements in Newton-Rapson Method for Nonlinear equations using Modified Adomain Decomposition Method", International Journal of Mathematical Analysis, Vol. 9, 2015, no. 39, 1919.1928.

[5] Muhammad Aslam Noor, Khalida Inayat Noor, Eisa Al-Said and Muhammad Waseem, "Some New Iterative methods for Nonlinear Equations, Hindawi Publishing Corporation.

[6] Okorie Charity Ebelechukwu, ben Obakpo Johnson, Ali Inalegwu Michael, akuji Terhemba fideli, "Comparison of Some Iterative methods of Solving Nonlinear Equations", International Journal of Theroretical and Applied Mathematics.

[7] Ogbereyivwe Oghovese, Emunefe O. John, "New Three-Steps Iterative Method for Solving Nonlinear Equations, IOSR Journal of Mathematics.

[8] Masoud Allame, Nafiseh azad, "A new method for solving nonlinear equations by Taylor expansion", Application of Mathematics and Computer engineering.

[9] Jishe Feng, "A New Two-step Method for solving Nonlinear equations", International Journal of Nonlinear Science", Vol.8(2009) No.1pp.40-44.

[10] Masoud Allame and Nafiseh Azad, "On Modified Newton Method for solving a Nonlinear Algebraic equation by Mid-Point”, World applied science Journal 17(12): 1546-1548, 2012.

[11] Mohammad Hani Almomani, Mahmoud Hasan Alrefaei, Shahd Al Mansour, "A method for selecting the best performance systems", International Journal of Pure and Applied Mathematics.

[12] Zolt 'an Kov'acs, "Understanding convergence and stability of the Newton-Raphson method", https://www.researchgate.net/publication/277475242.

[13] Fernando Brambila Paz, Anthony Torres Hernandez, Ursula Iturrarán-Viveros, Reyna Caballero Cruz, "Fractional Newton-Raphson Method Accelerated with Aitken's Method".

[14] Robin Kumar and Vipan, "Comparative Analysis of Convergence of Various Numerical Methods", Journal of Computer and Mathematical Sciences, Vol.6(6),290-297, June 2015, ISSN 2319-8133 (Online).

[15] Nancy Velasco, Dario Mendoza, Vicente Hallo, Elizabeth Salazar-Jácome and Victor Chimarro, "Graphical representation of the application of the bisection and secant methods for obtaining roots of equations using Matlab", IOP Conf. Series: Journal of Physics: Conf. Series 1053 (2018) 012026 doi :10.1088/1742-6596/1053/1/012026.

[16] Kamoh Nathaniel Mahwash, Gyemang Dauda Gyang, "Numerical Solution of Nonlinear Systems of Algebraic Equations", International Journal of Data Science and Analysis2018; 4(1): 20-23 http://www.sciencepublishinggroup.com/j/ijdsa. 
Applied Mathematics and Sciences: An International Journal (MathSJ), Vol. 6, No. 2/3, September 2019

[17] Farooq Ahmed Shah, and Muhammad Aslam Noor, "Variational Iteration Technique and Some Methods for the Approximate Solution of Nonlinear Equations", Applied Mathematics\& Information Sciences Letters An International Journal, http://dx.doi.org/10.12785/amisl/020303.

[18] M. A. Hafiz \& Mohamed S. M. Bahgat, "An Efficient Two-step Iterative Method for Solving System of Nonlinear Equations", Journal of Mathematics Research; Vol. 4, No. 4; 2012 ISSN 1916-9795 EISSN 1916-9809. 